\title{
Erratum to: Epigenetic histone modifications in a clinically relevant rat model of chronic ethanol-binge-mediated liver injury
}

Annayya R. Aroor • Ricardo J. Restrepo •

Kusum K. Kharbanda $\cdot$ Shivendra D. Shukla

Published online: 22 July 2014

(C) Asian Pacific Association for the Study of the Liver 2014

\section{Erratum to: Hepatol Int}

DOI 10.1007/s12072-014-9546-4

In the original publication of this article, Fig. $4 \mathrm{~b}$ legend H3TriMeK4 in the 'Y-axis' has been mentioned incorrectly and it should read as H3TriMeK9. The correct Fig. 4 is provided in this erratum.
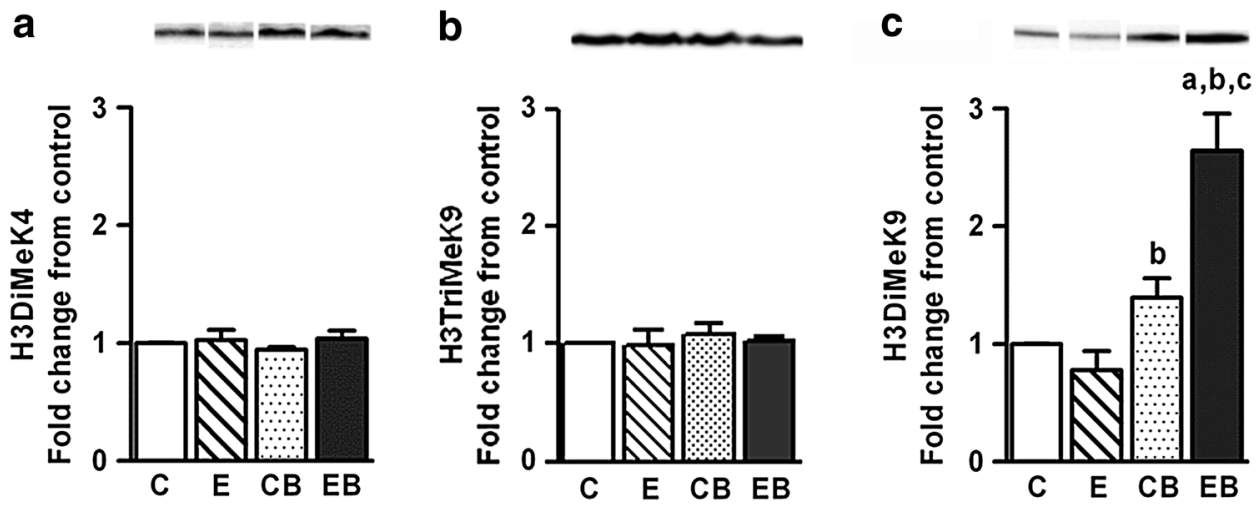

Fig. 4 Levels of dimethylated H3 K4, dimethylated histone H3 K9, and trimethylated histone $\mathrm{H} 3 \mathrm{~K} 9$ in different groups. The experimental protocol is similar to that in Fig. 1. Values are mean $\pm \mathrm{SE}$ $(n=4$ rats). $a$ Significant compared to the control group $(p<0.05)$;

$b$ significant compared to the chronic-ethanol group $(p<0.05)$; $c$ significant compared to the control-binge group. $C$ Control (pair fed), $E$ chronic ethanol, $C B$ control ethanol binge, $E B$ chronic ethanol binge

The online version of the original article can be found under doi:10.1007/s12072-014-9546-4.

\footnotetext{
A. R. Aroor · R. J. Restrepo · S. D. Shukla ( $\square)$

Department of Medical Pharmacology and Physiology,

University of Missouri School of Medicine, Columbia,

MO 65212, USA

e-mail: shuklasd@missouri.edu

K. K. Kharbanda

Veterans Affairs Nebraska-Western Iowa Health Care System,

Omaha, NE 68105, USA
} 